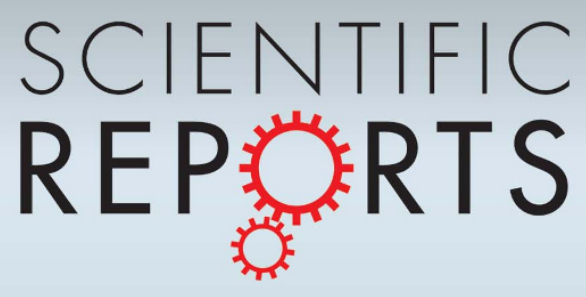

OPEN

SUBJECT AREAS:

CELL GROWTH

CELLULAR NEUROSCIENCE

BIOPHYSICS

DIFFERENTIATION

Received

13 May 2011

Accepted

14 October 2011

Published

11 November 2011

Correspondence and requests for materials should be addressed to

V.T. (torre@sissa.it)

* These authors equally contributed and their names are in alphabetical order

$\uparrow$ Present address: Leiden Institute of Chemistry, Molecular Genetics, Leiden, The Netherlands

\section{The elementary events underlying force generation in neuronal lamellipodia}

\author{
Ladan Amin ${ }^{1 *}$, Erika Ercolini ${ }^{1,2 *}$, Rajesh Shahapure ${ }^{1 * \dagger}$, Giacomo Bisson' $\&$ Vincent Torre $^{1,3}$ \\ ${ }^{1}$ Neurobiology Sector, International School for Advanced Studies (SISSA), IT-34136 Trieste, Italy, ${ }^{2}$ Cluster in Biomedicine (CBM), \\ Area Science Park Basovizza, IT-34012 Trieste, Italy, ${ }^{3}$ Italian Institute of Technology (IIT), SISSA Unit, IT-34 136 Trieste, Italy.
}

We have used optical tweezers to identify the elementary events underlying force generation in neuronal lamellipodia. When an optically trapped bead seals on the lamellipodium membrane, Brownian fluctuations decrease revealing the underlying elementary events. The distribution of bead velocities has long tails with frequent large positive and negative values associated to forward and backward jumps occurring in 0.1$0.2 \mathrm{~ms}$ with varying amplitudes up to $20 \mathrm{~nm}$. Jump frequency and amplitude are reduced when actin turnover is slowed down by the addition of $25 \mathrm{nM}$ Jasplakinolide. When myosin II is inhibited by the addition of $20 \mu \mathrm{M}$ Blebbistatin, jump frequency is reduced but to a lesser extent than by Jasplainolide. These jumps constitute the elementary events underlying force generation.

- orce generation is a fundamental process at the basis of cell motility ${ }^{1}$ allowing neurons to explore the - environment. Neuronal growth cones are the major motile structures located at the neurite tips ${ }^{2}$ and are composed of lamellipodia and filopodia ${ }^{3}$. Lamellipodia are extended structures, from which filopodia emerge with a finger-like shape $e^{4}$. Their motion is essential during morphogenesis and for neuronal differentiation when their exploratory motion allows neurons to find the appropriate synaptic connections. Force generation is thought to be originating from the progressive addition of actin molecules to the existing network of actin filaments ${ }^{5}$ and to be determined by the balance between actin polymerization and depolymerisation, modulated by controlling proteins ${ }^{6}$ and by chemical and mechanical receptors coupled to the cytoskeleton ${ }^{6-8}$. However, very little is known about the elementary events underlying force generation.

Actin polymerization has been primarily investigated in vitro by analysing the rate of elongation of isolated actin filaments. These investigations were performed with a low time resolution, often of the order of some tens of seconds and with a sensitivity of some hundreds of $\mathrm{nm}$, providing values for actin polymerization rate ranging between 11.6 and $38(1 / \mu \mathrm{M} \mathrm{s})^{9-12}$.

Previous investigations in vivo using Atomic Force Microscopy ${ }^{13}$ and opposing liquid flow ${ }^{14}$ were limited to a temporal resolution in the $100 \mathrm{~ms}$ range and sensitivity of 50-100 pN. These experimental limitations can be overcome by using optical tweezers ${ }^{15,16}$, providing a ms resolution and $\mathrm{pN}$ sensitivity. In order to detect small displacements in the order of $2-5 \mathrm{~nm}$ it is necessary to reduce all perturbations by minimizing mechanical vibrations and performing the experiments under remote conditions (see Methods). By using these procedures, we have previously shown that force generation is not a deterministic mechanism but follows a probabilistic process and that underlying dynamical events occur on different time scales varying from $100 \mathrm{~ms}$ to $5 \mathrm{~s}^{17}$.

For this study we have used optical tweezers to identify the elementary events underlying force generation. When an optically trapped bead seals on the lamellipodium membrane, Brownian fluctuations are drastically reduced revealing the fine structure of force generation: when a lamellipodium pushes a trapped bead, the autocorrelation function $\rho(t)$ of the bead position decays with multiple time constants up to $50 \mathrm{~ms}$, while during Brownian fluctuations $\rho(\mathrm{t})$ decays with a single time constant less than $1 \mathrm{~ms}$. The distribution of bead velocities has long tails with frequent large positive and negative values associated to forward and backward jumps occurring in 0.1-0.2 ms. These jumps have varying amplitudes up to $20 \mathrm{~nm}$ and their frequency and amplitude are reduced when actin turnover is slowed down by the addition of Jasplakinolide ${ }^{18}$ and when the action of myosin II is inhibited by the addition of Blebbistatin ${ }^{19,20}$. These jumps constitute the elementary events underlying force generation.

\title{
Results
}

Neurons from dorsal root ganglia (DRG) of P10-P12 rats were isolated and plated on poly-L-lysine-coated glass coverslips, positioned on the stage of an inverted microscope used for imaging and force measurement ${ }^{17}$ (see 
Methods). After 24 to 48 hours, lamellipodia emerged from DRG soma. Silica beads with a diameter of $1 \mu \mathrm{m}$ were trapped with an infrared (IR) optical tweezer in front of the lamellipodia (Fig. 1a and $\mathrm{f})$ : when the lamellipodia protruded and displaced the bead, the exerted force $F=\left(F_{x}, F_{y}, F_{z}\right)$ was measured with sub pN sensitivity at $10 \mathrm{kHz}$ resolution. The bead position $\boldsymbol{x}=(x, y, z)$ was measured with a quadrant position detector (QPD) using back focal plane (BFP) interferometry ${ }^{16,21}$. Lamellipodia grew by $1 \mu \mathrm{m}$ within $20-$ $30 \mathrm{~s}$ and displaced the beads trapped with a low $\left(k_{x}\right.$ and $k_{y}$ equal to $0.0155 \mathrm{pN} / \mathrm{nm}$, and $k_{z}$ equal to $\left.0.005 \mathrm{pN} / \mathrm{nm}\right)$ and a high stiffness $\left(k_{x}\right.$, and $k_{y}$ equal to $0.1 \mathrm{pN} / \mathrm{nm}$ and, $k_{z}$ equal to $0.03 \mathrm{pN} / \mathrm{nm}$; Fig. $1 \mathrm{a}-$ e). The QPD detects reliably lateral displacements less than $250 \mathrm{~nm}$ (see Methods) and bead displacements within this range were observed with the high trap stiffness. Often lamellipodia pushed the bead both laterally and axially (Fig. $1 \mathrm{f}-\mathrm{h}$ ) and recordings of the bead position became noisier (Fig. $1 \mathrm{k}$ ). In contrast, when adhesion forces caused the bead to seal onto the cellular membrane of retracting lamellipodia (Fig. 1i-j) Brownian fluctuations decreased (Fig. 1k). If growth cones were fixed with paraformaldehyde, suppressing all cellular motility, no noise increase was observed (see Supplementary Methods and Supplementary Fig. S1 online).

During adhesion, the variance $\left(\sigma_{\mathrm{x}}{ }^{2}, \sigma_{\mathrm{y}}{ }^{2}, \sigma_{\mathrm{z}}{ }^{2}\right)$ could decrease by $5-$ 10 times reaching values below $10 \mathrm{~nm}^{2}$ (Fig. $1 \mathrm{~m}$ ) so that the fine structure of force generation could be observed. The amplitude of the adhesion force $F_{a d}$ was measured as the maximal force before the bead returned into the trap ${ }^{22-24}$ (Fig. 11). Large values of $\boldsymbol{F}_{\boldsymbol{a d}}$ reduced more Brownian fluctuations (Fig. 1n). If $F_{a d}$ is larger than $30 \mathrm{pN}$, i.e. the maximal restoring force of the optical trap, when the
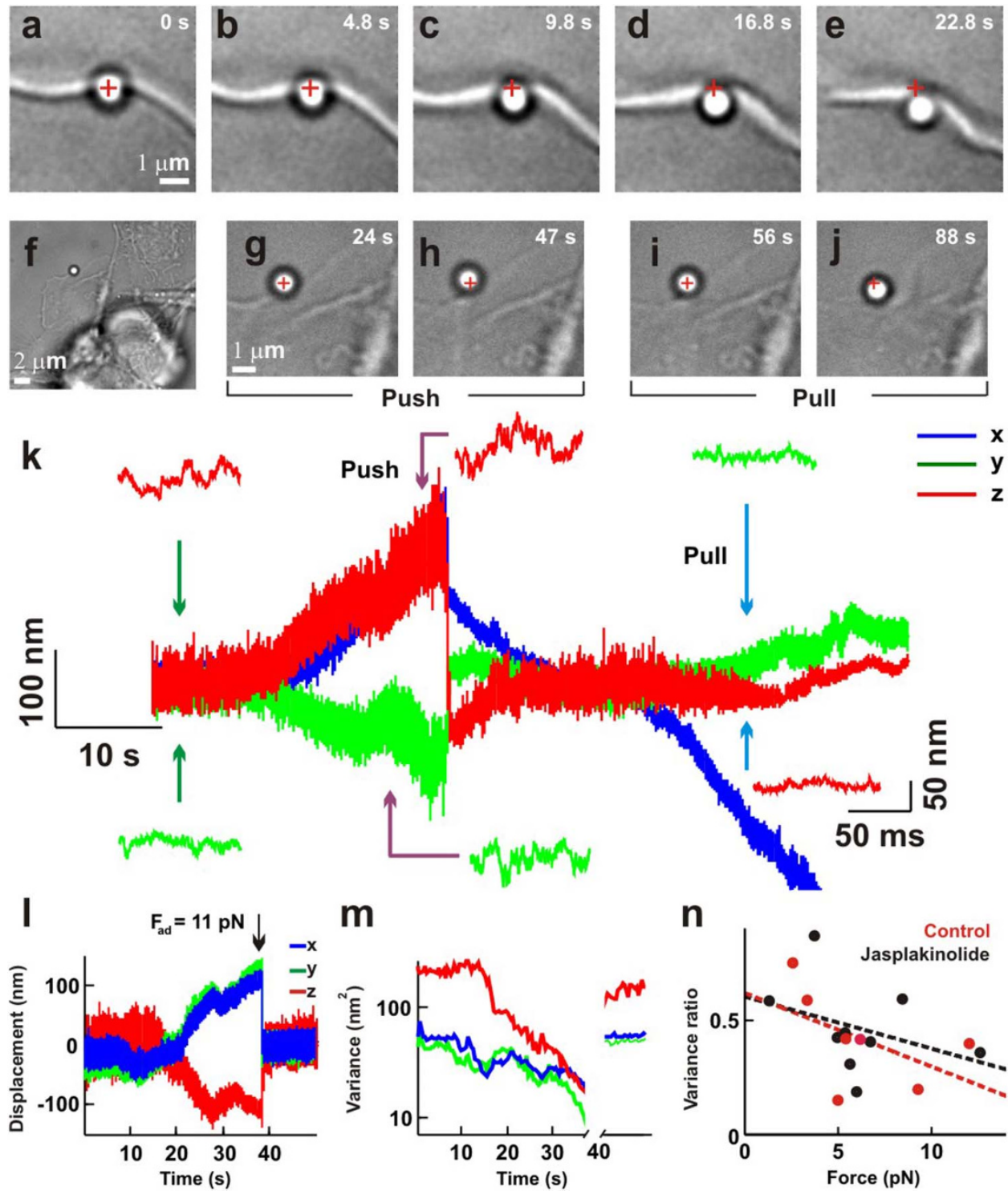

m

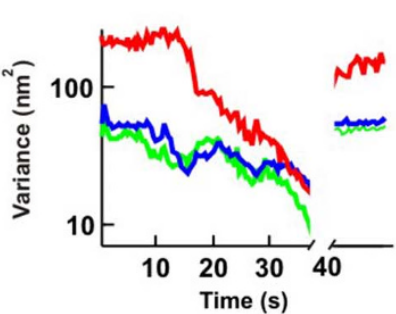

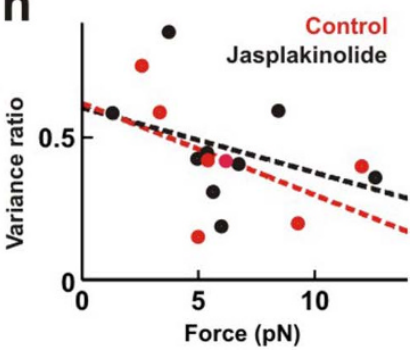

Figure 1 During a push, recordings of the bead position become noisier, but not during a pull. (a)-(e) The protruding leading edge of a lamellipodium pushes an optically trapped bead by $1 \mu \mathrm{m}$ within $25 \mathrm{~s}$. (f) A bead trapped in front of a lamellipodium emerging from the soma of a DRG neuron. (g)-(h) High resolution images during a push. At $24 \mathrm{~s}$ the bead is in the optical trap (g) and when the lamellipodium grows, it pushes the bead (47 s) displacing it both laterally and axially (h). (i) - (j) As in ( $\mathrm{g}-\mathrm{h}$ ) but during a pull. When the lamellipodium retracted, the bead returned inside the trap (56 $\mathrm{s})$. Following bead adhesion, the bead was pulled away from the trap $(88 \mathrm{~s})$. Crosses indicate the centre of the optical trap. $(\mathrm{k})$ The three components $(\mathrm{x}, \mathrm{y}, \mathrm{z})$ of the bead displacement. Insets highlight the increase of noise during the push (violet arrows), the decrease of noise during the pull (cyan arrows), and green arrows refer to Brownian fluctuations. (l) The three components (x,y,z) of bead displacement during adhesion and retraction in another experiment. At $38 \mathrm{~s}$ the bead returned into the trap and the adhesion force was measured $(11 \mathrm{pN}) .(\mathrm{m})$ Change of variance for the three components in (l). (n) Relation between fractional variance reduction and modulus of adhesion force in control conditions (red symbols) and in the presence of $25 \mathrm{nM}$ Jasplakinolide (black symbols). The red and black lines represent the linear fit in control conditions and Jasplakinolide, respectively. 
lamellipodium retracts or vigorously protrudes it could move the bead out of the trap. The bonding of a single integrin molecule to the lamellipodium leading edge is larger than $40 \mathrm{pN}^{25}$ and therefore, when integrin molecules cause adhesion, the bead is strongly attached and will follow the lamellipodium motion also out of the optical trap. During adhesion, the power spectrum density (PSD) of Brownian fluctuations was fitted by the sum of two or three Lorentzian distributions (see Supplementary Methods and Supplementary Fig. S1 online).

When actin turnover is reduced no noise increase is observed. During a push the variance of bead displacement increased by 2-4 times (Figs $1 \mathrm{k}$ and $2 \mathrm{a}$ ) possibly because of modifications of the trap stiffness, fluctuations of adhesion forces or properties of force generation. The addition of $100 \mathrm{nM}$ Jasplakinolide, known to reduce actin turnover ${ }^{18}$ almost completely abolished force generation, but a lower concentration of $25 \mathrm{nM}$ slowed down growth cone motion without blocking force generation. In the presence of $25 \mathrm{nM}$ Jasplakinolide, lamellipodia were still able to displace beads laterally, but no increase of variance was observed (compare Fig. 2a and $b$ ). We computed the relation between the lateral component of the force $\left(F_{x}\right.$ or $\left.F_{y}\right)$ and the associated changes of variance $\sigma_{1}{ }^{2}$. In the presence of $25 \mathrm{nM}$ Jasplakinolide, $\sigma_{1}^{2}$ never increased (grey black traces in Fig. 2d; $n=9$ ), but often decreased. On the contrary, in control conditions, $\sigma_{1}^{2}$ increased by $2-4$ times (red orange traces in Fig. $2 \mathrm{~d} ; \mathrm{n}=13$ ). During protrusion the maximal average velocity $\left\langle\mathrm{v}_{\max }>\right.$ was $50 \mathrm{~nm} / \mathrm{s}(\mathrm{n}=24)$, whereas in the presence of Jasplakinolide it was $35 \mathrm{~nm} / \mathrm{s}(\mathrm{n}=15)^{17}$. The mean value of the modulus of $\boldsymbol{F}_{\mathrm{ad}}$ in control conditions was $6.2 \pm 3.3 \mathrm{pN}(\mathrm{n}=7)$ and in the presence of $25 \mathrm{nM}$ Jasplakinolide was $6.1 \pm 3.1 \mathrm{pN}(\mathrm{n}=9)$ suggesting that adhesion between the bead and the lamellipodium is not affected by Jasplakinolide. Bead displacements and exerted forces were very similar in control conditions and in the presence of $25 \mathrm{nM}$ Jasplakinolide suggesting that the observed variance increase is not caused by local changes of trap stiffness but it is a genuine property of force generation.

When myosin II is inhibited force generation occurs with a reduced noise increase. The molecular motor myosin II plays an important role in force generation by speeding up actin filament disassembly ${ }^{26}$ and therefore we analysed the effect of Blebbistatin a well known inhibitor of myosin $\mathrm{II}^{19,20}$. The addition of $20 \mu \mathrm{M}$ Blebbistatin slowed down lamellipodia motion, but did not abolish force generation (Fig. 2c). During lateral push, however, $\sigma_{1}^{2}$ did not increase by more than $100 \%$ and in some cases ( 2 out of 6 ; blue traces in Fig. 2d) decreased, but not as observed with Jaslpakinolide (Fig. 2d).

The increase of noise is related to the contact area between the bead and the lamellipodium leading edge. The area in direct contact $A_{c}$ with a silica bead with a diameter of $1 \mu \mathrm{m}$ could vary from less than 0.1 to up to $1.5 \mu \mathrm{m}^{2}{ }^{17}$. This contact area mediates all mechanical interactions between the bead and force generation mechanisms inside lamellipodia. Therefore we have analysed the relation between $A_{c}$ and the amplitude of generated force $F$ and associated changes of variance $\sigma_{l}^{2}$. In control conditions, during a lateral push it is possible to measure reliably changes of $A_{c}$ and when $A_{c}$ increases (Fig. 3a) we have often (7 out of 10 experiments) observed a concomitant increase of $\mathrm{F}$ (Fig. 3b) and of $\sigma_{1}^{2}$ (Fig. 3c). When the bead went out of the optical trap (broken vertical line) the $A_{c}$ - obtained from videomicrographs - remained constant or increased while measurements of $\mathrm{F}$ and of $\sigma_{1}^{2}$ are not reliable. Therefore, the increase of $\sigma_{1}^{2}$ observed during lateral pushes is caused by the combined effect of force generation and of the associated increase of $A_{c}$. $A_{c}$ is measured through the objective of the microscope, viewing axially the lamellipodium, therefore we were not able to determined changes of $\mathrm{A}_{\mathrm{c}}$ during a vertical push. a

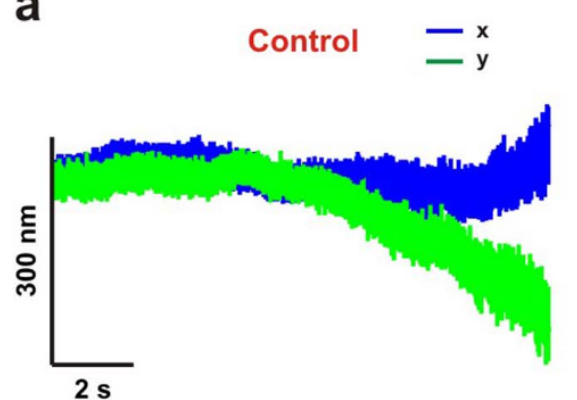

C

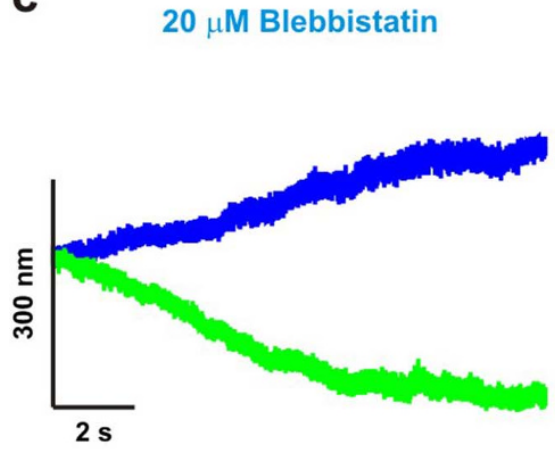

b

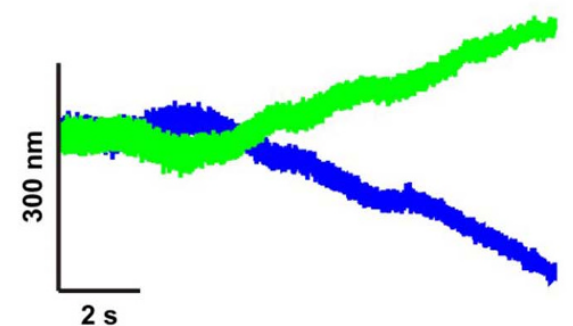

d

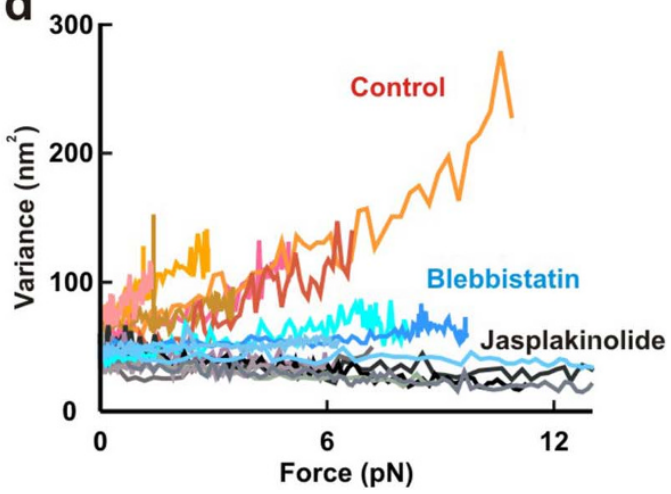

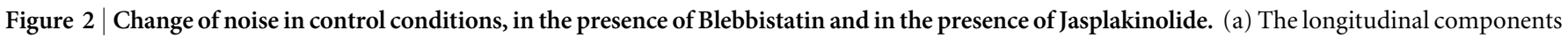
of the bead displacement during a lateral push in control conditions showing a clear noise increase. (b) As in (a) but in the presence of 25 nM

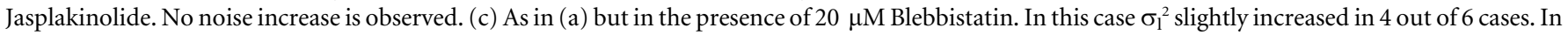

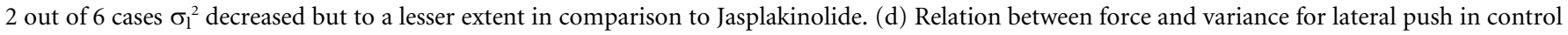
conditions (red shades), in the presence of $20 \mu \mathrm{M}$ Blebbistatin (blue shades) and $25 \mathrm{nM}$ Jasplakinolide (black shades). 


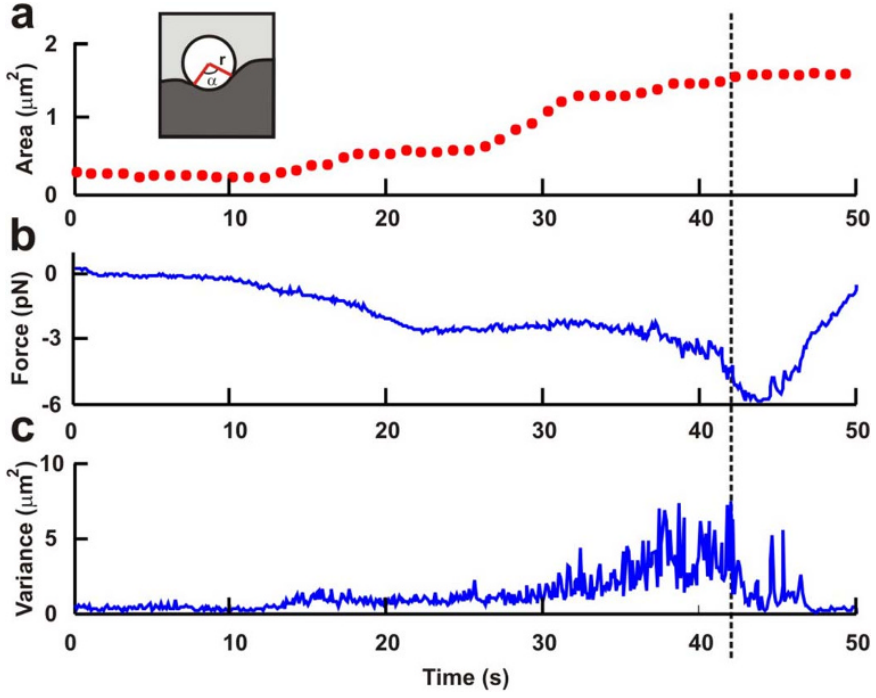

Figure 3 Concomitant change of variance and contact area during force generation. (a) Time evolution of estimated contact area $\mathrm{A}_{c}$ (see Ref. 17) between the bead and the lamellipodium leading edge, $A_{c}$, during a push. $A_{c}$ at frame $i, A_{c}(i)$, is calculated as $A_{c}(i)=2 \pi\left[1-\cos \left(\alpha_{i} / 2\right)\right] r^{2}$, where $\alpha_{i}$ is the angle corresponding to the arc of the bead in close contact with the leading edge of the lamellipodium and $r$ is the bead radius, as shown in the inset, representing a lamellipodium pushing the trapped bead. (b) Concomitant time evolution of the force exerted by the lamellipodium during the push analyzed in (a). (c) Concomitant time evolution of the variance during the push analyzed in (a). The broken vertical line indicates the time when the bead is pushed out of the trap.
Properties of noise during pushes. Following adhesion, in several experiments $(n=4$ in control conditions; $n=6$ with Jasplakinolide; $\mathrm{n}=5$ with Blebbistatin) the variance of the axial component, $\sigma_{\mathrm{a}}{ }^{2}$, decreased to less than $6 \mathrm{~nm}^{2}$ (Fig. 4a) and subsequently, when the lamellipodium pushed the bead, $\sigma_{\mathrm{a}}{ }^{2}$ increased and fluctuations with novel properties appeared.

Visual inspection indicated the existence of rapid discontinuities, i.e. of jumps. Therefore, we computed the bead velocity v (Fig. 4b), by convolution of bead position with the derivative of a Gaussian function, $\left(-\mathrm{t} /\left((2 * \pi)^{1 / 2} * a^{3}\right) \exp \left(-\mathrm{t}^{2} / 2 * a^{2}\right)\right.$ with a value of $a$ varying from 0.2 to $0.4 \mathrm{~ms}$. During Brownian fluctuations, velocities had a Gaussian distribution (Fig. 4c) but not during a push (Fig. 4d): indeed their distribution had a central lobe fitted by a Gaussian distribution, but had also long tails with large positive and negative values. These sudden changes of velocity correspond to forward $\left(j^{+}\right)$ and backward $\left(j^{-}\right)$jumps. Similar tails, but less pronounced, could be detected also when force generation was not preceded by adhesion. During Brownian fluctuations the autocorrelation function $\rho_{\mathrm{zz}}(\mathrm{t})$ of bead displacement decayed with a single time constant $\theta$ of $0.64 \pm$ $0.12 \mathrm{~ms}(\mathrm{n}=20)$, but during pushes $\rho_{\mathrm{zz}}(\mathrm{t})$ decayed with multiple time constants varying from less than $1 \mathrm{~ms}$ up to $50 \mathrm{~ms}$ (Fig. 4e). During force generation, fluctuations in three coordinates $(\mathrm{x}, \mathrm{y}, \mathrm{z})$ were more correlated: during Brownian fluctuations the cross-correlation $\rho_{\mathrm{zl}}(\mathrm{t})$ between $\mathrm{z}$ and one lateral component ( $\mathrm{x}$ or $\mathrm{y}$ ) decayed with a time constant $\theta$ of $0.62 \pm 0.15 \mathrm{~ms}$ (black traces in Fig. $4 \mathrm{~g}$ ), but during a push the value of $\theta$ increased to $6.0 \pm 1.4 \mathrm{~ms}$ (red traces in Fig. $4 \mathrm{~g}$ ). The increase of the time constant $\theta$ of $\rho_{z z}(t)$ and $\rho_{z l}(t)$ observed during a push was attenuated by $25 \mathrm{nM}$ Jasplakinolide (black trace in Fig. $4 \mathrm{f}$ and red traces in Fig. 4h).

All these observations indicate that: i- fluctuations observed during pushes do not originate from thermal motion but are caused by the randomness of the elementary events underlying force generation; ii - molecular mechanisms underlying force generation are a

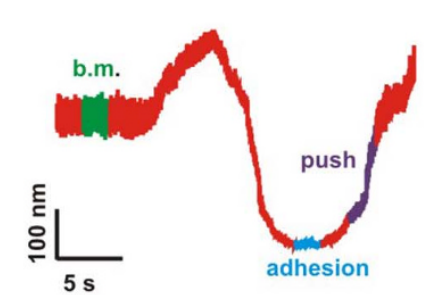

e

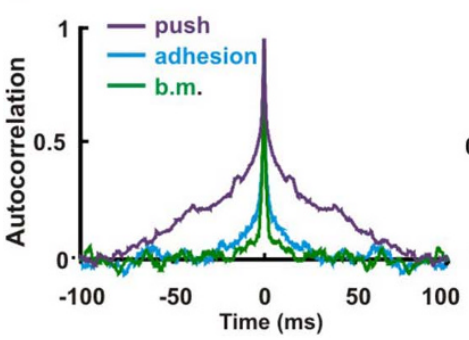

b
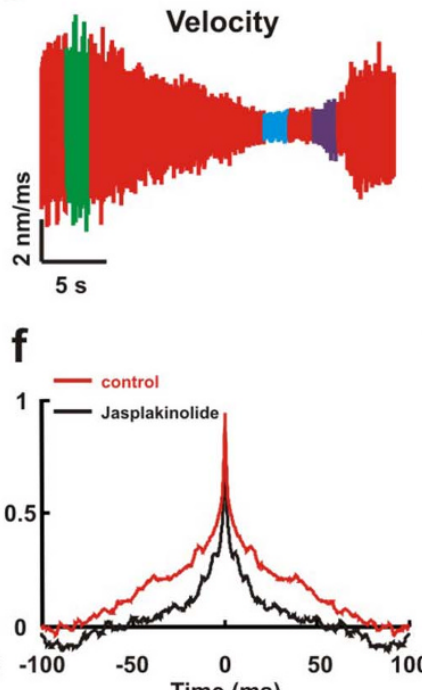

C

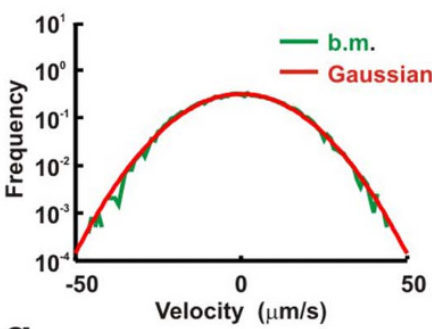

g

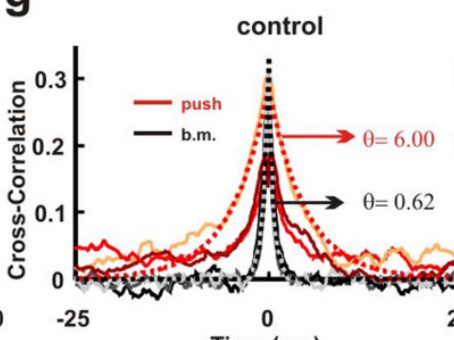

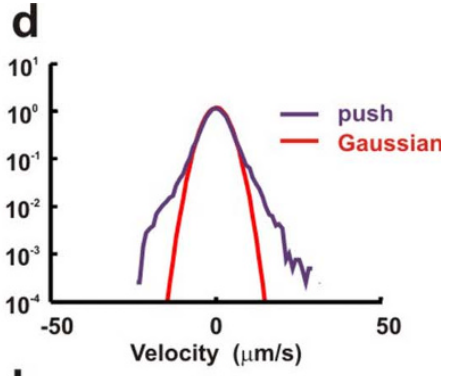

h

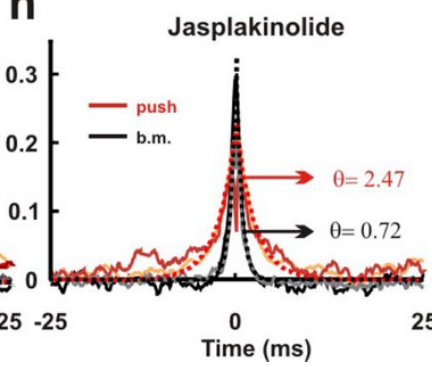

Figure $4 \mid$ During pushes the autocorrelation function $\rho(t)$ of bead position decays with multiple time constants and the distribution of bead velocities has long tails. (a) The z component of the bead displacement during Brownian fluctuations (b.m), adhesion and push. (b) Velocity of bead displacement in (a). (c) Distribution of velocities during Brownian fluctuations shown in (b). A Gaussian function (red line) fits perfectly the experimental distribution. (d) As in (c) during the push shown in (b). (e) Autocorrelation function of vertical bead displacement $\rho_{\mathrm{zz}}(\mathrm{t})$, during Brownian fluctuations, adhesion, and push after high pass filtering with a cut-off frequency at $1 \mathrm{~Hz}$ (see Supplementary Methods and Supplementary Fig. S2 online). $\rho_{\mathrm{zz}}(\mathrm{t})$ decays with a time constant $\theta$ equal to $0.64 \mathrm{~ms}$ during b.m. but, during pushing, it has multiple time constants up to $50 \mathrm{~ms}$. (f) The effect of $25 \mathrm{nM}$ Jasplakinolide on $\rho_{\mathrm{zz}}(\mathrm{t})$, during pushing (black). The longest time constant of the auto-correlation decreases to $20 \mathrm{~ms}$ (red trace). (g) Cross-correlation $\rho_{\mathrm{zx}}(\mathrm{t})$ during b.m. (black shades) and during a push (red shades). $\rho_{\mathrm{zx}}(\mathrm{t})$ decays with a time constant $\theta$ equal to $0.62 \mathrm{~ms}$ during b.m. and increases to $6.0 \mathrm{~ms}$ during a push. (h) The effect of $25 \mathrm{nM}$ Jasplakinolide on $\rho_{\mathrm{zx}}(\mathrm{t})$ during pushing. $\rho_{\mathrm{zx}}(\mathrm{t})$ decays with a time constant $\theta$ equal to $0.72 \mathrm{~ms}$ during b.m. and increases to $2.47 \mathrm{~ms}$ during a push. 
spatially coherent and structured; iii- force generation is characterized by jumps.

Jumps underlie force generation. Among the vast repertoire of algorithms used to detect jumps, we adopted a nonlinear diffusion filtering ${ }^{27,28}$, approximating the original data (grey traces in Fig. 5a, b and c) with a smooth piece-wise function (red lines) interrupted by $j^{+}$ or $\mathrm{j}^{-}$jumps (black vertical segments). This algorithm depends on two parameters (see Methods): the contrast $\lambda$, related to the smallest detectable jump, and the scale $\tau$ determining the temporal window. In order to establish our sensitivity and to determine the values of $\lambda$ and $\tau$ we attached a silica bead to the bottom of a coverslip which was moved by a piezo manipulator. When the variance of displacement fluctuations of the stuck bead was $3.8 \mathrm{~nm}^{2}$, as during adhesion (Fig. 4a), with the values of $0.5 \mathrm{~nm}$ and $0.1 \mathrm{~ms}$ for $\lambda$ and $\tau$ respectively, we could detect jumps of $2 \mathrm{~nm}$. With these values of $\lambda$ and $\tau$, the algorithm detected jumps primarily during pushes (Fig. $5 \mathrm{a}$, $\mathrm{b}$ and $\mathrm{c}$ ).

Immediately after adhesion during the push, (Figs $4 \mathrm{a}$ and $5 \mathrm{a}$ ), we detected forward $j^{+}$and backward $j^{-}$jumps ranging from 2 to $20 \mathrm{~nm}$
(Fig. 5d). Jumps were observed only when lamellipodia pushed the bead, but very rarely when beads sealed on the lamellipodia membrane and retracted, suggesting that jumps do not reflect unspecific attachment/detachment events between the lamellipodium and the substratum and/or between the actin network and the membrane. Jumps lower than $2 \mathrm{~nm}$ could not be detected because of noise limitations. These jumps appear to be the elementary events underlying force generation in neuronal lamellipodia.

Distributions of jumps amplitude in control conditions (Fig. 5d) were fitted by the exponential distributions $A_{+} e^{-(j+/ j+*)}$ and A $\mathrm{e}^{-(\mathrm{j}-\mathrm{j}-*)}$ with values of $5.2 \pm 1.3$ and $4.9 \pm 1.2 \mathrm{~nm}$ for the mean size of positive $\mathrm{j}^{+*}$ and negative jumps $\mathrm{j}^{-*}(n=4)$. In the presence of $25 \mathrm{nM}$ Jasplakinolide (Fig. 5c) smaller jumps (Fig. 5f) ranging from 2 to $8 \mathrm{~nm}$ were detected $(\mathrm{n}=6)$ and jump distributions were fitted by the same exponential distributions but with lower values of $j^{+}$ and $j^{-}$equal to $2.4 \pm 0.3$ and $2.2 \pm 0.4 \mathrm{~nm}$, similar in size to the polymerization step size $(2.7 \mathrm{~nm})$. If Jasplakinolide is reducing jump frequency by stiffening the connection between the lamellipodium and the bead, it is expected also to modify the adhesion force between the bead and the lamellipodium, but this was not observed

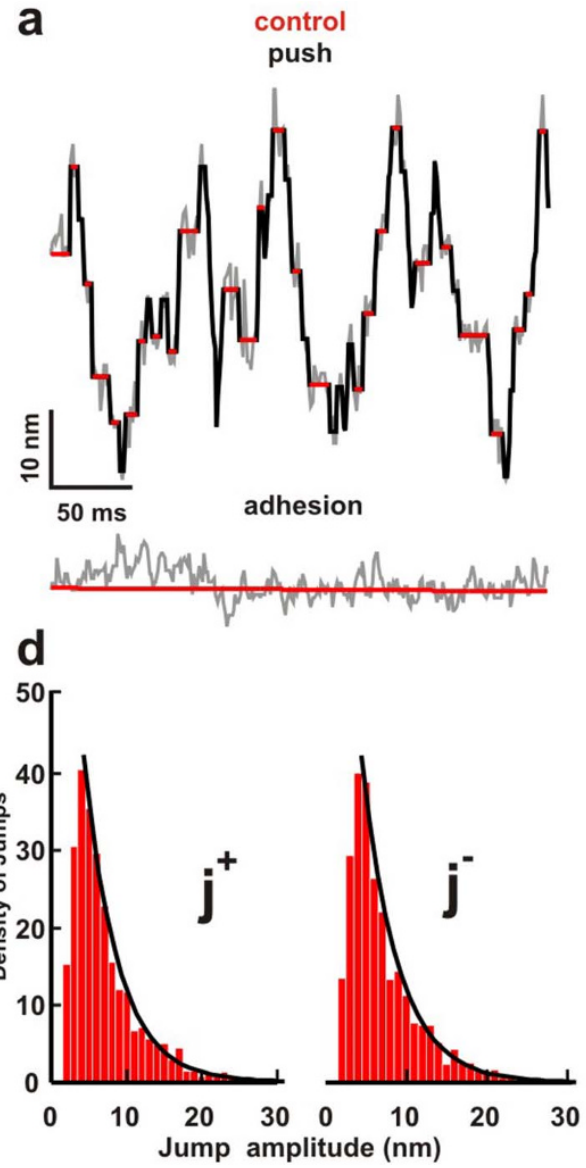

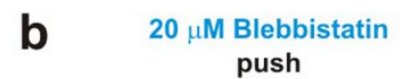
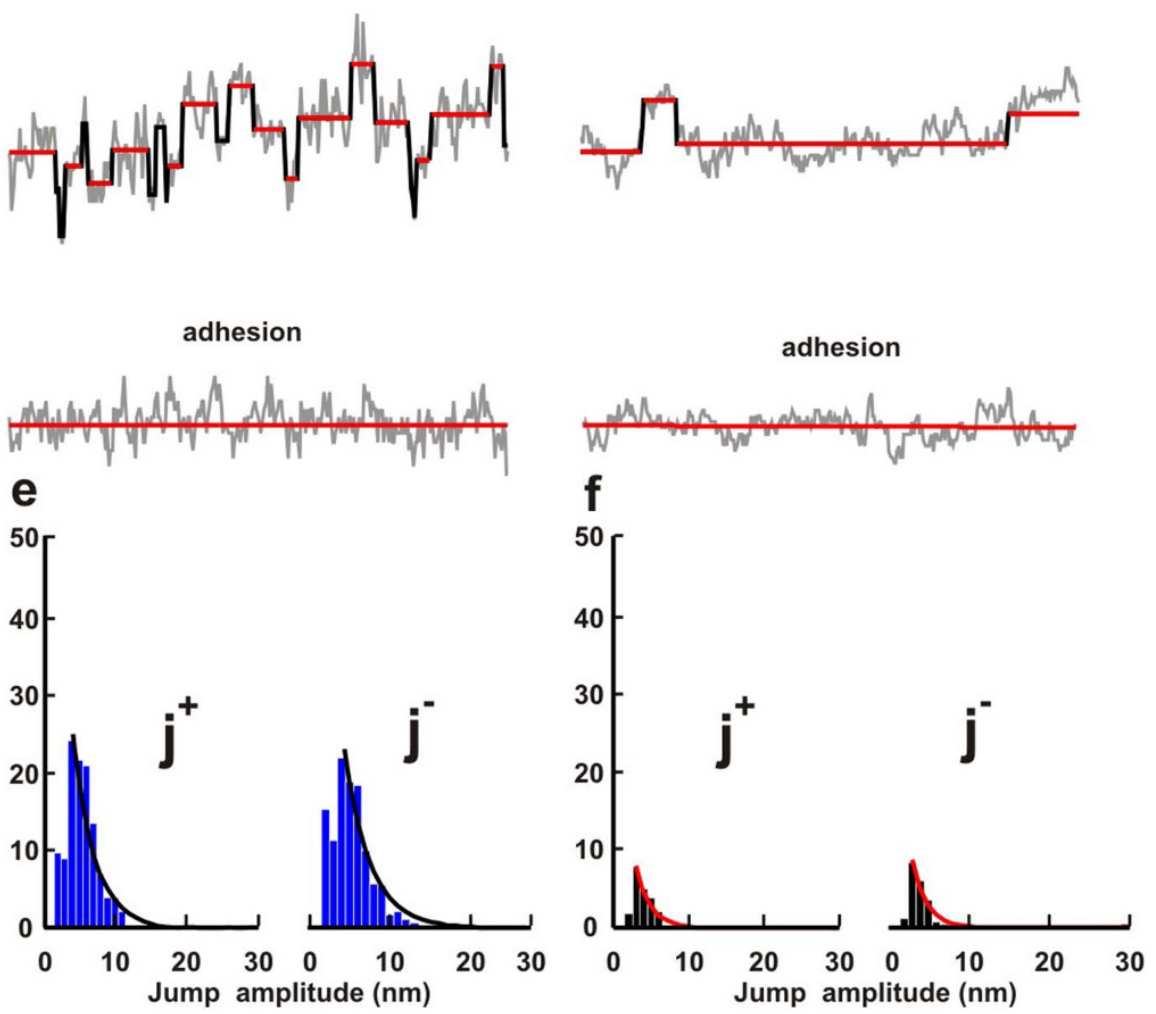

Figure $5 \mid$ Forward and backward jumps are the elementary events underlying force generation. (a) Magnification of the $\mathrm{z}$ component of Fig. 4 a during adhesion and push. Original traces in grey were filtered by the non linear diffusion algorithm (see Methods) providing a smooth component (red curves) and jumps (in black). Very few jumps were detected during adhesion but they could be observed very often during a push. (b) Magnification of the $\mathrm{z}$ component during adhesion and push in the presence of $20 \mu \mathrm{M}$ Blebbistatin. The original traces (in gray) were filtered as in (a). Jumps with smaller amplitude than in control conditions were detected. (c) As in (a) and (b) in the presence of $25 \mathrm{nM}$ Jasplakinolide. In this case jumps with an amplitude smaller than the amplitude obtained both in control conditions and in the presence of Blebbistatin were detected. (d)-(f) Density of upward $j^{+}$and downward $j^{-}$jumps during push in control conditions (d), in the presence of Blebbistatin (e), and Jasplakinolide (f). These distributions of jump amplitude were fitted (black lines in (d) and (e), red lines in (f) ) - for values of $\mathrm{j}^{+}$and $\mathrm{j}^{-}$larger than $2 \mathrm{~nm}$-by the exponential distributions $\mathrm{A}_{+} \mathrm{e}^{-(\mathrm{j}+\mathrm{j}+*)}$ and $A_{-} e^{-(j-/ j-*)}$. The fitting was performed with the values of of 129 and 128 events/s for the jump frequency of positive and negative jumps, $A_{+}$and $A_{-}$, respectively, and 5 and $4.8 \mathrm{~nm}$ for the mean size of positive and negative jumps, $\mathrm{j}^{+*}$ and $\mathrm{j}^{-*}$, respectively (d). In the presence of Blebbistatin the values of $\mathrm{A}_{+}, \mathrm{A}_{-}, \mathrm{j}^{+*}$ and $\mathrm{j}^{-*}$ were 87 and 80 events/s and 3.5 and $3.3 \mathrm{~nm}$, respectively (e). In the presence of Jasplakinolide the values of $\mathrm{A}_{+}, \mathrm{A}_{-}, \mathrm{j}^{+*}$ and $\mathrm{j}^{-*}$ were 44 and 50 events/s and 2.5 and $2.3 \mathrm{~nm}$, respectively (f). 
experimentally (Fig. 1n). Therefore, the effect of Jasplakinolide on jump frequency is likely to be caused by a reduced actin turnover. In the presence of $20 \mu \mathrm{M}$ Blebbstatin (Fig. 5b) detected jumps have an amplitude ranging from 2 to $15 \mathrm{~nm}(\mathrm{n}=5)$. Distributions of jumps amplitude in the presence of Blebbistatin were fitted by exponential distributions with values of $\mathrm{j}^{+*}$ and $\mathrm{j}^{-*}$ equal to $3.4 \pm 0.9$ and $3.2 \pm 0.8 \mathrm{~nm}$. Jumps in the presence of Blebbistatin occurred with a frequency $50 \%$ lower than in control conditions. Therefore Jasplakinolide decreased the jumps frequency and their amplitude more than Blebbistatin.

The detection and measurements of jumps in the presence of noise is a difficult (ill-posed) problem ${ }^{29}$ that should not be underestimated. Therefore, in order to show that jumps are real and not artifacts of used algorithms, it is needed to verify that large values of bead velocity v (Fig. 6a) were coincident with jumps (Fig. 6b) detected by the non linear diffusion algorithm (see vertical lines in panels $6 \mathrm{a}$ and $6 \mathrm{~b}$ ). Given the time series of bead position $\left(x_{n} n=1, . . N\right)$ the computation of the instantaneous velocity does not require any parameter, because the velocity is equal to $\left(\mathrm{x}_{\mathrm{n}+1}-\mathrm{x}_{\mathrm{n}}\right) / \Delta \mathrm{t}$, but two parameters are involved in the nonlinear diffusion algorithm $(\lambda$ and $\tau)$. In order to establish co-localization in a quantitative way, large values of $\mathrm{v}$ were assumed to be those belonging to the tails of the velocity distribution outside the Gaussian function fitting its central lobe (see Fig. $4 \mathrm{~d}$ ) and these values of $\mathrm{v}$ co-localized in a time window $\Delta \mathrm{t}$ of less than $0.3 \mathrm{~ms}$ with detected jumps. The analysis of the Rate of True Positive (RTP) and of False Positive co-localization ${ }^{30}$ (Fig. 6c) indicates that for $\Delta t$ equal to $100 \mu$ s, i.e. the used sampling interval, RTP is larger than $80 \%$ and becomes close to $100 \%$ for $\Delta$ t equal to $300 \mu$ s. This analysis indicates that jumps detected by the non linear diffusion algorithm co-localize very precisely with large values of bead velocity.

Jumps were clearly detected when force generation developed following adhesion. i.e. when Brownian fluctuations were reduced (Fig. 5). However, more often force generation developed without being preceded by bead adhesion (Fig. 7a). Therefore we asked whether it was possible to determine the existence of jumps also when force generation did not follow bead adhesion. As force generation is characterized by a large value of the autocorrelation function (Fig. 4) we computed for all three components $x, y$ and $z$, $\rho_{x x}(t), \rho_{y y}(t), \rho_{z z}(t)$. During Brownian fluctuations $\rho_{i i}(t)$ are exponentially distributed with a value of $\tau$ less than $1 \mathrm{~ms}$, but during push $\rho_{\mathrm{ii}}(\mathrm{t})$ become broader decaying with several time constants (Fig. 4). Therefore we computed the integral $\mathrm{C}_{\mathrm{i}}(\mathrm{t})$ of $\rho_{\mathrm{ii}}(\mathrm{t})$ at each time and force generation was identified to occur when $\mathrm{C}_{\mathrm{i}}(\mathrm{t})$ increased by at least 10 times (Fig. 7b). Under these circumstances the variance of bead fluctuations at the peak of force generation was significantly higher than during Brownian fluctuations (Fig. 7c). During this phase the central lobe of the distributions of $\mathrm{dx} / \mathrm{dt}$, dy/dt and $\mathrm{dz} / \mathrm{dt}$ was fitted by a Gaussian function, but tails corresponding to large positive and negative velocities were detected (see arrows in Fig. $7 \mathrm{~d}-$ f) indicating the existence of forward and backward jumps. Jumps detected by the non linear diffusion algorithm during these events (Fig. $7 \mathrm{~g}-\mathrm{i}$ ) have amplitudes ranging up to $20 \mathrm{~nm}$, as those detected after adhesion (Fig. 5).

If jumps are the elementary events underlying force generation their sum must be close to the observed net protrusion. Therefore we compared the net protrusion $\operatorname{Prot}(\Delta \mathrm{t})$ in the time window $\Delta \mathrm{t}$ with the sum of all forward jumps $\left(\Sigma_{\Delta t} j^{+}\right)$minus the sum of all backward jumps $\left(\Sigma_{\Delta \mathrm{t}} \mathrm{j}^{-}\right)$occurring in $\Delta \mathrm{t}: \mathrm{P}(\Delta \mathrm{t})$ (red line) was very similar to $\Sigma_{\Delta t} j^{+}-\Sigma_{\Delta t} j^{-}$(black line in Fig. 8a and b) in control conditions as well as in the presence of Jasplakinolide. We next asked whether force generation developed by an increase of the frequency of jumps, i.e. $\mathrm{A}_{+}$and $\mathrm{A}_{-}$or by their mean amplitude, i.e. $\mathrm{j}^{+*}$ and $\mathrm{j}^{-*}$. Therefore we estimated $A_{+}, A_{-}, j^{+*}$ and $j^{-*}$ in $0.5 \mathrm{~s}$ intervals during force generation: force generation developed by a combination of an increase of jumps frequency and of their mean values. The observation that forward and backward jumps sum to net protrusion is an additional test for internal consistency of the used procedure for jump detection, providing further support that jumps are the elementary events underlying force generation.

\section{Discussion}

The results of the present manuscript show that force generation in neuronal lamellipodia of rat DRG neurons, is composed by a

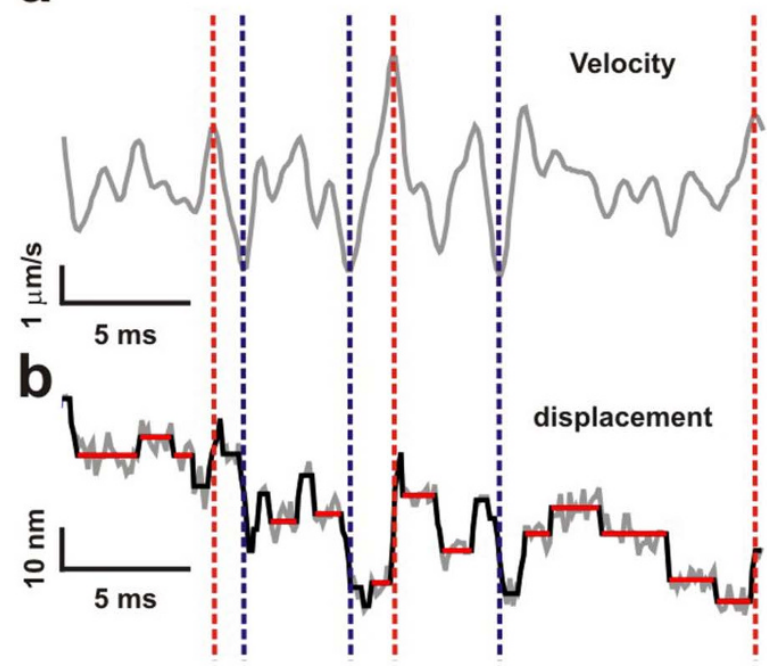

C

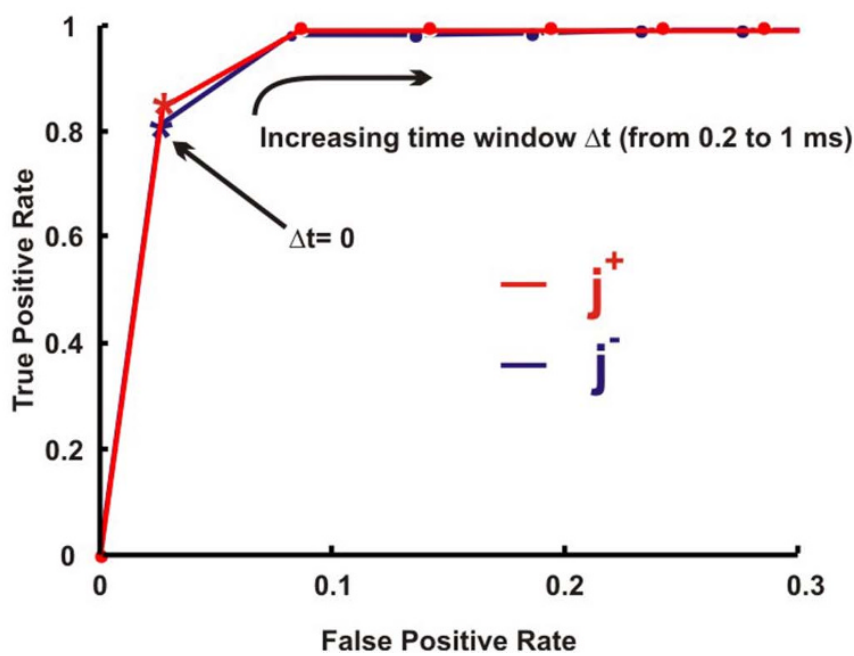

Figure 6 Colocalization of jumps and large values of bead velocity. (a) Bead velocity during a push obtained by the convolution of the bead displacement with the derivative of a Gaussian function $\left(-\mathrm{t} /(2 * \pi)^{1 / 2} * a^{2}\right) \exp \left(-\mathrm{t}^{2} / 2^{*} a^{2}\right)$ with $a=0.1 \mathrm{~ms}$. A jump and a large value of $\mathrm{v}$ colocalize if they occur in a time window $\Delta \mathrm{t}$ of less than $0.3 \mathrm{~ms}$. Large values of $\mathrm{v}$ were those belonging to the long tails of velocity distribution. These velocities had an absolute value larger than 3 times the standard deviation of the Gaussian fitting the central lobe of the velocity distribution (see Fig. 4d). (b) Jumps detected by nonlinear diffusion in the same portion of the push shown in (a), where velocity was computed. Original trace, gray; smoothed component, red; detected jumps, black. Red and black dotted lines highlight colocalization of positive $\mathrm{j}^{+}$and negative $\mathrm{j}^{-}$jumps, respectively with large values of $\mathrm{v}$. (c) Rate of True Positive colocalization and of False Positive colocalization for increasing values of $\Delta \mathrm{t}$ from 0 to $1 \mathrm{~ms}$, Asterisks represent the classifiers in which positive (red) and negative (black) jumps colocalize exactly with large values of $\mathrm{v}^{30}$. 
a
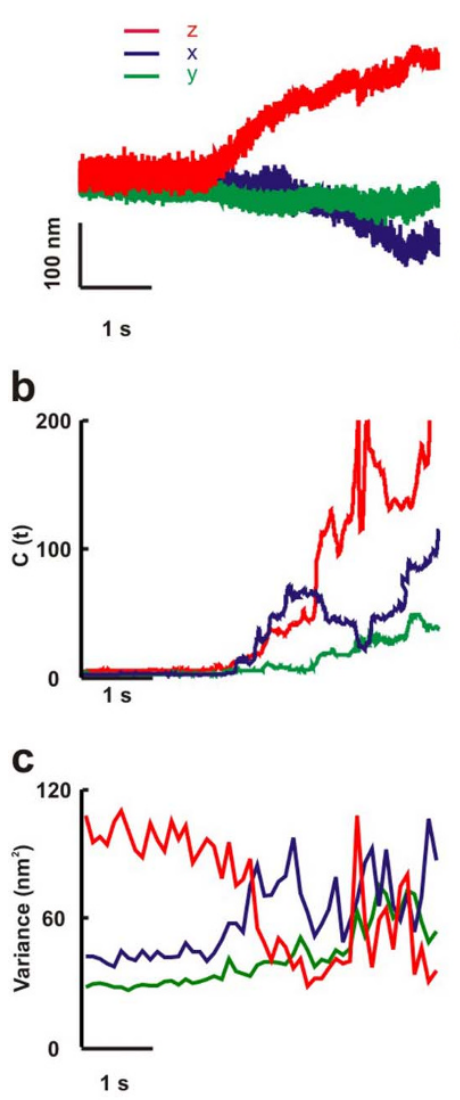

d

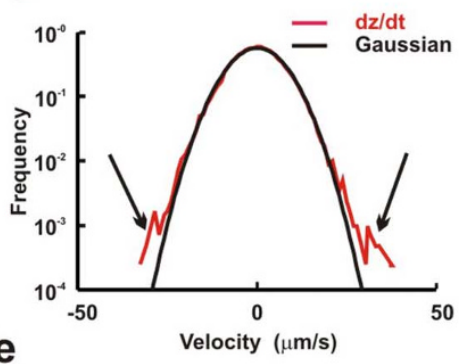

e
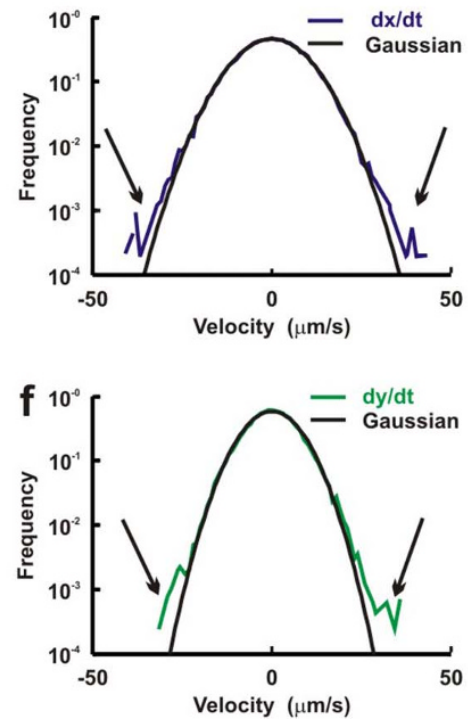

g

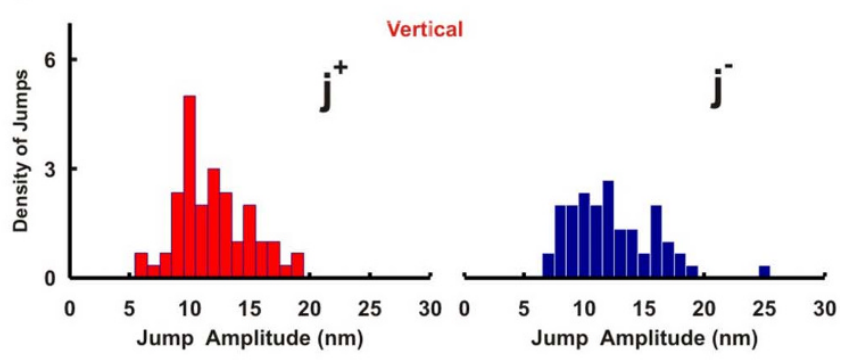

h
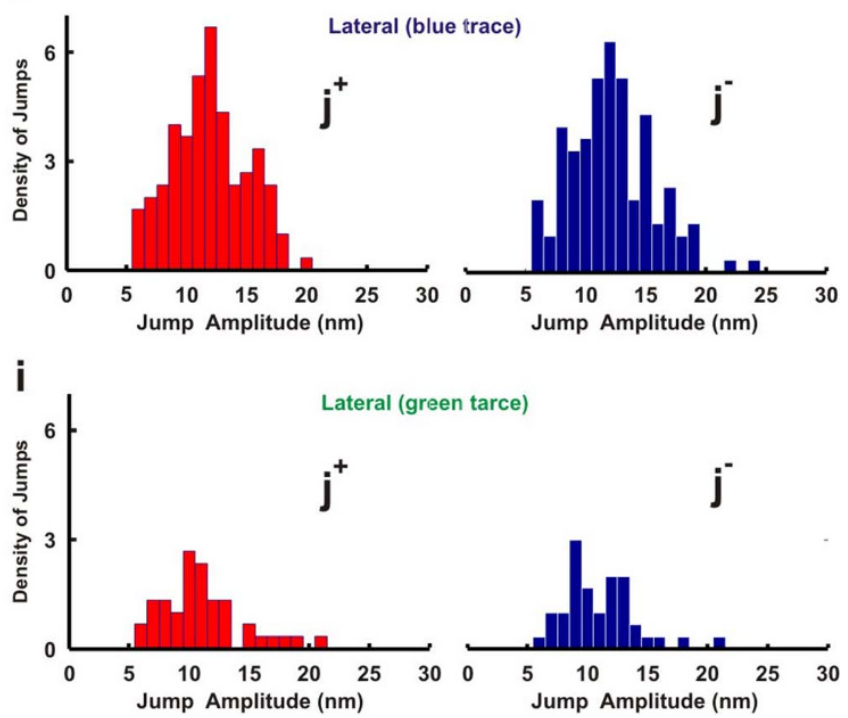

Figure 7 Characterization of force generation during a push in the absence of adhesion. Episodes of force generation were identified as increases of at least 10 times of the integral $C_{i}(t)$ of $\rho_{i i}(t), i=x, y, z$. (a) The three components $(x, y, z)$ of the bead displacement during a push. (b) Integral $C_{x}(t)(b l u e)$, $\mathrm{C}_{\mathrm{y}}(\mathrm{t})\left(\right.$ green), and $\mathrm{C}_{\mathrm{z}}(\mathrm{t})(\mathrm{red})$ of the autocorrelation function $\rho_{\mathrm{xx}}(\mathrm{t}), \rho_{\mathrm{yy}}(\mathrm{t})$, and $\rho_{\mathrm{zz}}(\mathrm{t})$ of each of the three components of the bead displacements shown in (a). (c) Change in time of the bead displacement variance for the three components in (a). Variance computed in time windows of $0.1 \mathrm{~s}$ after high pass filtering at $1 \mathrm{~Hz}$. (d)-(f) Distribution of values of velocity dz/dt (d), dx/dt (e), and dy/dt (f), during force generation shown in (a). The black line represents the Gaussian fit to the distribution. The arrows highlight the tails associated to forward and backward jumps. (g) - (i) Density of upward $j^{+}$(red histograms) and downward $\mathrm{j}^{-}$(blue histograms) jumps during the push shown in (a) for the $\mathrm{z}(\mathrm{g}), \mathrm{x}(\mathrm{h})$, and $\mathrm{y}$ (i) component, respectively.

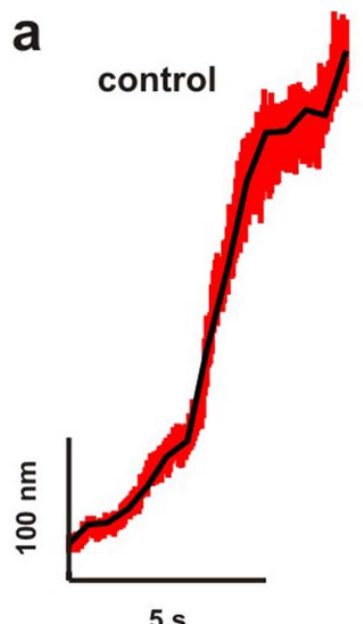

b Jasplakinolide

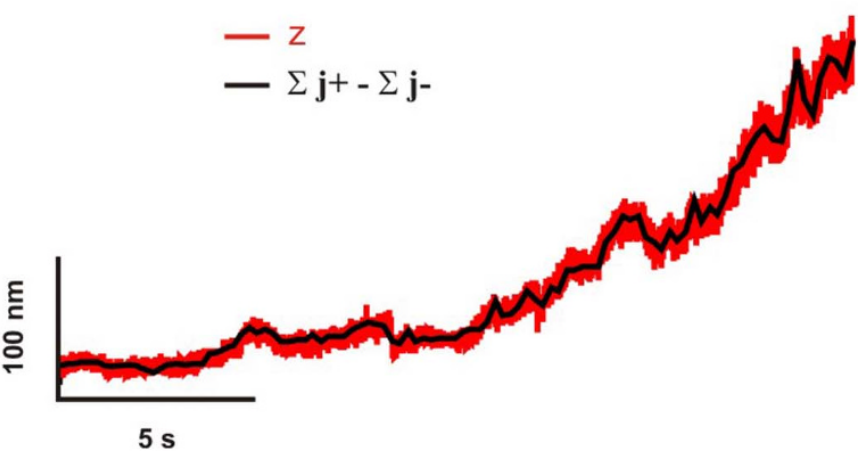

Figure $8 \mid$ The sum of forward and backward jumps is equal to the net protrusion. (a) Bead vertical displacement during a push (red line) in control conditions. The black line represents the sum of all forward jumps $\left(\Sigma_{\Delta t} j^{+}\right)$minus the sum of all backward jumps $\left(\Sigma_{\Delta t} j^{-}\right)$occurring in the time window $\Delta \mathrm{t}=0.5 \mathrm{~s}$, calculated over the whole push. (b) As in (a) in the presence of $25 \mathrm{nM}$ Jasplakinolide. 
elementary events corresponding to forward and backward jumps. These jumps have an amplitude ranging from 2 to $20 \mathrm{~nm}$ and have varying orientation in the $3 \mathrm{D}$ space. These jumps are not observed when growth cones were fixed with paraformaldehyde, suppressing all cellular motility (Supplementary Methods and Supplementary Fig. S1 online) and their amplitude and frequency were reduced by treating growth cones with $25 \mathrm{nM}$ Jasplakinolide (Fig. 5). Jumps detected by the nonlinear diffusion algorithm colocalize with high values of the instantaneous bead velocity (Fig. 6) and the net protrusion of lamellipodia is the net sum of forward and backward jumps (Fig. 8). For all these reasons, jumps - here described-are neither artifacts of the detection procedure nor are caused by changes of properties of the optical trap. Detected jumps represent the elementary events underlying force generation in DRG lamellipodia.

Force generation occurs at different rates. At the slowest rate the lamellipodium leading edge advances smoothly with forward and backward jumps (Fig. 5f) with an amplitude similar in size to the mean polymerization step size $(2.7 \mathrm{~nm})$ observed during actin filament polymerization ${ }^{31,32}$ suggesting that actin monomers are added one by one to the existing network of filaments. At the fastest rate (Fig. 5d) larger jumps are observed and they could result from the insertion of small actin oligomers ${ }^{33}$ and by the occurrence of a burst of actin polymerization in a single or neighboring actin filaments. Oligomers of 5-10 actin molecules are present in lamellipodia, primarily as a result of actin filaments depolymerization and could be used for actin filament assembly, as in yeast Saccharomyes cervisiae ${ }^{33}$. Experimental determinations of free G-actin in lamellipodia varied from 1-3 $\mu \mathrm{M}^{9-12}$ to values 100 higher $^{34}$. In vitro determination of actin polymerization rates provides values ranging from 11.6 to $38(1 / \mu \mathrm{M} \mathrm{s})^{9-12}$, but the bulk turnover of actin subunits is $100-200$ times faster in cells than in vitro ${ }^{1,35,36}$. Therefore, the occurrence of a fast and vigorous polymerization rate of a single actin filament is possible. As the estimated density of actin filaments impinging upon the leading edge of a lamellipodium is between 100 and 200 per $\mu \mathrm{m}^{2}{ }^{13,32}$ a burst of polymerization of several actin filaments and an appropriate spatial environment could also produce a discrete forward step of $10-20 \mathrm{~nm}$ of the lamellipodium leading edge. When actin turnover is reduced by Jasplakinolide ${ }^{18}$ and when myosin II is inhibited by Blebbistatin ${ }^{19,20}$, force generation still occurs but at a slower rate (Fig. 5e and f). Jumps produced by DRG lamellipodia do not have a discrete amplitude as observed in the actin-based movement of the bacterium Listeria monocytogenes, with a predominant jump size of $5.4 \mathrm{~nm}^{37}$.

Force generation in lamellipodia depends on several factors such as the availability of actin monomers/oligomers, the presence of molecular motors such as myosin II and a large variety of controlling proteins ${ }^{6}$. This complexity is at the basis of the observed dynamics, reminiscent of self organized criticality ${ }^{38}$.

\section{Methods}

Neuron preparation. Wistar rats (P10-P12) were anaesthetized with CO2 and sacrificed by decapitation in accordance with the Italian Animal Welfare Act. The Ethics Committee of the International School for Advanced Studies (SISSA-ISAS) has approved the protocol (Prot.n. 2189-II/7). Dorsal Root Ganglia (DRG) were incubated with trypsin $(0.5 \mathrm{mg} / \mathrm{ml}$, Sigma-Aldrich, Milan, Italy), collagenase (1 mg/ $\mathrm{ml}$, Sigma-Aldrich), and DNase $(0.1 \mathrm{mg} / \mathrm{ml}$, Sigma-Aldrich) in $5 \mathrm{ml}$ Neurobasal medium (Gibco, Invitrogen, Milan, Italy) in a shaking bath $\left(37^{\circ} \mathrm{C}, 35-40\right.$ minutes). DRGs were mechanically dissociated, centrifuged at $300 \mathrm{rpm}$, resuspended in culture medium and plated on poly-L-lysine-coated $(0.5 \mu \mathrm{g} / \mathrm{ml}$, Sigma-Aldrich $)$ coverslips. Cells were incubated for 24 to 48 hours followed by the addition of nerve growth factor $(50 \mathrm{ng} / \mathrm{ml}$; Alomone, Israel) before the measurements.

Optical tweezer set-up. The optical tweezers set-up was built as previously described ${ }^{17,39}$. The dish containing the differentiating neurons and the beads (PSI$1.0 \mathrm{NH} 2$, G.Kisker GbR, Steinfurt Germany) was placed on the microscope stage which could be moved by a 3 axis piezoelectric nanocube (17 MAX 301, Melles Griot Inc., USA). The temperature of the dish was kept at $37^{\circ} \mathrm{C}$ by a Peltier device. The bead position was determined in the $\mathrm{x}, \mathrm{y}$ and $\mathrm{z}$ plane with a lateral and axial accuracy of 2 and $5 \mathrm{~nm}$ respectively, which was obtained from the analysis of the interference between forward scattered light from the bead and unscattered light ${ }^{16,21}$. The back focal plane of the condenser was imaged onto a QPD (C5460SPL 6041, Hamamatsu, Milan, Italy) and the light was converted to differential outputs digitized at $10 \mathrm{kHz}$ and low pass filtered at $5 \mathrm{kHz}$. Both the lateral and axial trap stiffness, $\mathbf{k} x y=(k x, k y)$ and $k z$, respectively, as well as the detector sensitivity were calibrated using the power spectrum method ${ }^{16}$ with voltage signals filtered and digitized at $5 \mathrm{kHz}$. In order to reduce and possibly avoid all mechanical perturbations affecting the measurement of $\mathbf{x}=(x, y, z)$, the optical tweezers set-up was kept in an isolated and sound-proof room and the scientists performing the experiments, controlled all operations remotely from a separate room. In order to have good mechanical stability it was necessary to position all power supplies of used equipment in a separate room and to avoid flying cables by properly securing them. In this way we reduced perturbations, which could have affected previous investigations.

Jumps determination by non linear diffusion filtering. In order to detect jumps, we used an algorithm based on non linear diffusion ${ }^{27,28}$. After selecting the part of the trace of interest, the original signal was approximated with a smooth piece-wise function where the discontinuities, i.e. rapid and large changes, were identified as jumps. The non-linear diffusion is an iterative process based on the choice of two parameters: the contrast $\lambda$, related to the minimal jump amplitude detected, and the scale $\tau$, determining the temporal window of jumps. The values of $\lambda$ and $\tau$ were set equal to $0.5 \mathrm{~nm}$ and $0.1 \mathrm{~ms}$, respectively, so to detect 2 ground truth jumps (see Supplementary Methods and Supplementary Fig. S3 online). The algorithm is based on the Toolbox of Frederico D'Almeida (see http://www.mathworks.com/ matlabcentral/fileexchange/3710-nonlinear-diffusiontoolbox).

We compared the detection of jumps using the same values of $\lambda$ and $\tau$ from traces obtained in different conditions (Brownian fluctuation recording, adhesion, and push; see Supplementary Methods and Supplementary Fig. S3 online), but scaled so to have the same width of the central lobe of the velocity distribution. During pushes, jumps were detected with a rate about 4 times higher than in the other conditions. Therefore, if the variance of Brownian fluctuations of the trapped bead decreases to or below $4 \mathrm{~nm}^{2}$, the overall system can detect reliably $2 \mathrm{~nm}$ jumps.

1. Pollard, T. D. \& Borisy, G. G. Cellular motility driven by assembly and disassembly of actin filaments. Cell 112, 453-465 (2003).

2. Bray, D., Thomas, C. \& Shaw, G. Growth cone formation in cultures of sensory neurons. Proc. Natl. Acad. Sci. U. S. A 75, 5226-5229 (1978).

3. Huber, A. B., Kolodkin, A. L., Ginty, D. D. \& Cloutier, J. F. Signaling at the growth cone: ligand-receptor complexes and the control of axon growth and guidance. Annu. Rev. Neurosci. 26, 509-563 (2003).

4. Landis, S. C. Neuronal growth cones. Annu. Rev. Physiol. 45, 567-580 (1983).

5. Pollard, T. D., Blanchoin, L. \& Mullins, R. D. Molecular mechanisms controlling actin filament dynamics in nonmuscle cells. Annu. Rev. Biophys. Biomol. Struct. 29, 545-576 (2000).

6. Song, H. J. \& Poo, M. M. The cell biology of neuronal navigation. Nat. Cell Biol. 3, E81-E88 (2001).

7. Gallo, G. \& Letourneau, P. C. Neurotrophins and the dynamic regulation of the neuronal cytoskeleton. J. Neurobiol. 44, 159-173 (2000).

8. Gordon-Weeks, P. R. Microtubules and growth cone function. J. Neurobiol. 58 70-83 (2004)

9. Pollard, T. D. \& Mooseker, M. S. Direct measurement of actin polymerization rate constants by electron microscopy of actin filaments nucleated by isolated microvillus cores. J. Cell Biol. 88, 654-659 (1981).

10. Pollard, T. D. Rate constants for the reactions of ATP- and ADP-actin with the ends of actin filaments. J. Cell Biol. 103, 2747-2754 (1986).

11. Quinlan, M. E., Heuser, J. E., Kerkhoff, E. \& Mullins, R. D. Drosophila Spire is an actin nucleation factor. Nature 433, 382-388 (2005).

12. Michelot, A. et al. Actin-filament stochastic dynamics mediated by ADF/cofilin Curr. Biol. 17, 825-833 (2007).

13. Prass, M., Jacobson, K., Mogilner, A. \& Radmacher, M. Direct measurement of the lamellipodial protrusive force in a migrating cell. J. Cell Biol. 174, 767-772 (2006).

14. Bohnet, S., Ananthakrishnan, R., Mogilner, A., Meister, J. J. \& Verkhovsky, A. B. Weak force stalls protrusion at the leading edge of the lamellipodium. Biophys. J. 90, 1810-1820 (2006)

15. Bustamante, C., Macosko, J. C. \& Wuite, G. J. L. Grabbing the cat by the tail: Manipulating molecules one by one. Nat Rev Mol Cell Biol 1, 130-136 (2000)

16. Neuman, K. C. \& Block, S. M. Optical trapping. Rev. Sci. Instrum. 75, 2787-2809 (2004).

17. Shahapure, R. et al. Force generation in lamellipodia is a probabilistic process with fast growth and retraction events. Biophys. J. 98, 979-988 (2010).

18. Bubb, M. R., Spector, I., Beyer, B. B. \& Fosen, K. M. Effects of jasplakinolide on the kinetics of actin polymerization. An explanation for certain in vivo observations. J. Biol. Chem. 275, 5163-5170 (2000).

19. Straight, A. F. et al. Dissecting temporal and spatial control of cytokinesis with a myosin II inhibitor. Science 299, 1743-1747 (2003).

20. Kovacs, M., Toth, J., Hetenyi, C., Malnasi-Csizmadia, A. \& Sellers, J. R. Mechanism of blebbistatin inhibition of myosin II. Journal of Biological Chemistry 279, 35557-35563 (2004).

21. Gittes, F. \& Schmidt, C. F. Interference model for back-focal-plane displacement detection in optical tweezers. Opt. Lett. 23, 7-9 (1998). 
22. Simpson, K. H., Bowden, G., Hook, M. \& Anvari, B. Measurement of adhesive forces between individual Staphylococcus aureus MSCRAMMs and proteincoated surfaces by use of optical tweezers. J. Bacteriol. 185, 2031-2035 (2003).

23. Jass, J. et al. Physical properties of Escherichia coli P pili measured by optical tweezers. Biophys. J. 87, 4271-4283 (2004).

24. Knoner, G. et al. Mechanics of cellular adhesion to artificial artery templates. Biophys. J. 91, 3085-3096 (2006).

25. Helenius, J., Heisenberg, C. P., Gaub, H. E. \& Muller, D. J. Single-cell force spectroscopy. Journal of Cell Science 121, 1785-1791 (2008).

26. Medeiros, N. A., Burnette, D. T. \& Forscher, P. Myosin II functions in actinbundle turnover in neuronal growth cones. Nat Cell Biol 8, 215-226 (2006).

27. Perona, P. \& Malik, J. Scale-Space and Edge-Detection Using Anisotropic Diffusion. Ieee Transactions on Pattern Analysis and Machine Intelligence 12, 629-639 (1990).

28. Weickert, J. Applications of nonlinear diffusion in image processing and computer vision. ACTA MATHEMATICA UNIVERSITATIS COMENIANAE $\mathbf{7 0}$ 33-50 (2001).

29. Bertero, M., Poggio, T. A. \& Torre, V. Ill-Posed Problems in Early Vision. Proc IEEE 76, 869-889 (1988).

30. Fawcett, T. An introduction to ROC analysis. Pattern Recognition Letters 27, 861-874 (2006).

31. Egelman, E. H. The Structure of F-Actin. Journal of Muscle Research and Cell Motility 6, 129-151 (1985).

32. Abraham, V. C., Krishnamurthi, V., Taylor, D. L. \& Lanni, F. The actin-based nanomachine at the leading edge of migrating cells. Biophys. J. 77, 1721-1732 (1999).

33. Okreglak, V. \& Drubin, D. G. Loss of Aip1 reveals a role in maintaining the actin monomer pool and an in vivo oligomer assembly pathway. J. Cell Biol. 188, 769-777 (2010).

34. Koestler, S. A. et al. F- and G-actin concentrations in lamellipodia of moving cells. PLoS. One. 4, e4810 (2009).

35. Pantaloni, D., Le Clainche, C. \& Carlier, M. F. Mechanism of actin-based motility. Science 292, 1502-1506 (2001).

36. Small, J. V., Stradal, T., Vignal, E. \& Rottner, K. The lamellipodium: where motility begins. Trends Cell Biol. 12, 112-120 (2002).
37. Kuo, S. C. \& McGrath, J. L. Steps and fluctuations of Listeria monocytogenes during actin-based motility. Nature 407, 1026-1029 (2000).

38. Cardamone, L., Laio, A., Torre, V., Shahapure, R. \& DeSimone, A. Cytoskeletal actin networks in motile cells are critically self-organized systems synchronized by mechanical interactions. Proceedings of the National Academy of Sciences of the United States of America 108, 13978-13983 (2011).

39. Cojoc, D. et al. Properties of the force exerted by filopodia and lamellipodia and the involvement of cytoskeletal components. PLOS ONE 2, e1072 (2007).

\section{Acknowledgments}

We thank Walter Vanzella (Glance Vision Technologies s.r.l.) for computational support. This work was funded by the European Commission under the Seventh Framework Programme: Project CP - FP 214566-2 NanoScale and Project n.229375 SMD.

\section{Author contributions}

L.A., E.E., and R.S. equally contributed and their names are in alphabetical order. V.T. conceived the project and designed the experiments. L.A., E.E., and R.S. performed the experiments and analyzed the data. L.A., E.E., R.S., and G.B. contributed to reagents/ materials/analysis tools. V.T., L.A., E.E., and R.S. wrote the paper.

\section{Additional information}

Supplementary information accompanies this paper at http://www.nature.com/ scientificreports

Competing financial interests: The authors declare no competing financial interests. License: This work is licensed under a Creative Commons Attribution-NonCommercial-ShareAlike 3.0 Unported License. To view a copy of this license, visit http://creativecommons.org/licenses/by-nc-sa/3.0/

How to cite this article: Amin, L., Ercolini, E., Shahapure, R., Bisson, G. \& Torre, V. The elementary events underlying force generation in neuronal lamellipodia. Sci. Rep. 1, 153; DOI:10.1038/srep00153 (2011). 